\title{
BMJ Open Feasibility, psychological outcomes and practical use of a stress-preventive leadership intervention in the workplace hospital: the results of a mixed-method phase-II study
}

\author{
Felicitas Stuber (D) , ${ }^{1}$ Tanja Seifried-Dübon (D) , ${ }^{1}$ Elena Tsarouha, ${ }^{2}$ \\ Zahra Rahmani Azad, ${ }^{1}$ Rebecca Erschens (D) , ${ }^{1}$ Ines Armbruster, ${ }^{1}$ \\ Susanne Schnalzer, ${ }^{3}$ Nadine Mulfinger, ${ }^{4}$ Andreas Müller, ${ }^{5}$ Peter Angerer, ${ }^{6}$ \\ Madeleine Helaß, ${ }^{7}$ Imad Maatouk, ${ }^{7}$ Christoph Nikendei, ${ }^{7}$ Sascha Ruhle (D) , \\ Bernd Puschner (D) , ${ }^{9}$ Harald Gündel, ${ }^{4}$ Monika A Rieger, ${ }^{2}$ Stephan Zipfel, ${ }^{1}$ \\ Florian Junne ${ }^{1,10}$
}

To cite: Stuber F, SeifriedDübon T, Tsarouha E, et al. Feasibility, psychological outcomes and practical use of a stress-preventive leadership intervention in the workplace hospital: the results of a mixed-method phase-II study. BMJ Open 2022;12:e049951. doi:10.1136/ bmjopen-2021-049951

- Prepublication history and additional supplemental material for this paper are available online. To view these files, please visit the journal online (http://dx.doi.org/10.1136/ bmjopen-2021-049951).

Received 08 February 2021 Accepted 20 December 2021

D) Check for updates

(c) Author(s) (or their employer(s)) 2022. Re-use permitted under CC BY-NC. No commercial re-use. See rights and permissions. Published by BMJ.

For numbered affiliations see end of article.

Correspondence to

Felicitas Stuber;

felicitas.stuber@med.uni-

tuebingen.de

\section{ABSTRACT}

Objectives Hospitals are psychologically demanding workplaces with a need for context-specific stresspreventive leadership interventions. A stress-preventive interprofessional leadership intervention for middle management has been developed. This phase-II study investigates its feasibility and outcomes, including work-related stress, well-being and transformational leadership.

Design This is a mixed-methods study with three measure points (T0: baseline, $\mathrm{T} 1$ : after the last training session, T2: 3-month follow-up). Additionally, focus groups were conducted to assess participants' change in everyday work.

Setting A tertiary hospital in Germany.

Participants $\mathrm{N}=93$ leaders of different professions. Intervention An interactive group setting intervention divided in five separate sessions ((1) self-care as a leader, (2) leadership attitudes and behaviour, (3) motives, needs and stressors of employees, (4) strengthen the resource 'team', (5) reflection and focus groups). The intervention was conducted between June 2018 and March 2020 in $k=5$ runs of the intervention.

Outcome measures Feasibility and acceptance were measured with a self-developed intervention specific questionnaire. Psychological outcomes were assessed with the following scales: work-related strain with the Irritation Scale, well-being with the WHO-5 Well-being Index and transformational leadership with the Questionnaire of Integrative Leadership.

Results After the intervention at $\mathrm{T} 2$, over $90 \%$ of participants reported that they would recommend the intervention to another coworker $(92.1 \%, n=59)$ and all participants $(n=64)$ were satisfied with the intervention and rated the intervention as practical relevant for their everyday work. Participants' self-rated cognitive irritation was reduced, whereas their well-being and transformational leadership behaviour were improved over time. Focus group discussions revealed that participants

\section{Strengths and limitations of this study}

The concept of the stress-preventive leadership intervention was developed with the help of interprofessional mental health experts and based on a systematic review of leadership interventions in the healthcare sector.

- The intervention was piloted with an extensive mixed methods approach.

- To assess psychological outcomes, standardised questionnaires were used.

- The focus group interviews were evaluated with a standardised method of qualitative content analysis.

- Due to the uncontrolled study design, only intrapersonal change could be recorded over time.

implemented intervention contents successfully in their everyday work.

Conclusions This intervention was feasible and showed first promising intraindividual changes in psychological outcomes. Participants confirmed its practical relevance. As a next step, the intervention will be evaluated as part of a multicentre-randomised controlled trial within the project SEEGEN (SEElische GEsundheit am Arbeitsplatz KrankeNhaus).

\section{INTRODUCTION}

Hospitals are demanding workplaces characterised by high demand and low control ${ }^{12}$ and high effort-reward imbalance. ${ }^{3} 4$ Workrelated strain, reduced well-being and even symptoms of mental illnesses such as depression are common in hospital workers. ${ }^{56}$ Also sick days caused by mental illnesses are higher in the healthcare sector than in other fields of work in Germany. ${ }^{7}$ Since the beginning of 
2020, hospital workers are additionally burdened by the acute health crisis due to the Covid-19 pandemic which is related negatively to their well-being. ${ }^{8}$ Reducing psychological strain in hospital employees is of great importance for both the individual and the society as well-being of hospital workers is related to their intention to leave, ${ }^{9}$ to productivity, ${ }^{10}$ to patient safety ${ }^{11-13}$ and ultimately to public welfare. Thus, stress-preventive measures in hospitals are urgently needed.

One stress-preventive measure at the workplace can be constructive leadership behavior. ${ }^{14}$ Transformational leadership is a constructive change-oriented leadership behaviour, which helps leaders to create a stress-preventive work structure and culture for followers by fostering innovations, supporting the development of team spirit and performance growth, focusing on followers' individuality, providing a vision and being a role model. ${ }^{15} 16$ Concerning the influence of leadership behaviour on followers' well-being, transformational leadership has been extensively examined. Transformational leadership has positive effects on followers increased well-being and reduced affective symptoms such as burnout. ${ }^{17}$

But previous research on leadership interventions showed mixed results. Whereas Tsutsumi ${ }^{18}$ postulated that leadership interventions show a short-term effect on followers' mental health, Kuehnl et $a l^{19}$ could not report effects of leadership interventions on followers' wellbeing. With regard to the healthcare sector the evaluation of leadership interventions with a focus on followers' or leaders' mental health have been almost neglected so far. In a recent systematic review, we found only a small number of leadership intervention studies in the health care sector. ${ }^{20}$ Within the small study sample four studies assumed a significant positive trend for either leaders, ${ }^{, 21} 22$ or followers' mental health ${ }^{2324}$ two studies could not identify a trend difference ${ }^{2526}$ and one study only reported an association between the personal competence and work strain/psychological impairment via regression analysis. ${ }^{27}$

Due to the scarcity of data on leadership interventions in the workplace hospital, little is known about change potential, feasibility and acceptance of leadership interventions and about leaders' subjective experience in their everyday work after intervention participation. Therefore, we developed an interprofessional stress-preventive leadership intervention for the middle management in the workplace hospital based on evidence-based concepts and needs analysis through semistructured interviews. The leadership intervention aims to target hospital leaders' own strain management, their competence to design working conditions and their constructive leadership behaviour.

We focused on leaders' own strain management since leaders' in the healthcare sector can experience high psychological strain due to their demanding work tasks. ${ }^{28}$ Moreover, leaders' own strain is negatively related to their leadership behaviour and their workplace relationships. ${ }^{29}$ This holds especially true for leaders of middle management as they work close to the base and experience rather high psychosocial demands. Strengthening leaders' own strain management skills could heighten their awareness of stress in the workplace hospital and make them role models in stress management, which could help to reduce strain among their followers.

By familiarising leaders' with work-related stress models for example, effort-reward imbalance, ${ }^{4}$ leaders could be strengthen in their ability to design followers' working conditions stress-preventivly. Since working conditions such as role clarity or predictability mediate the effect of leadership behaviour on followers' well-being, ${ }^{30}$ an improvement in working conditions could further promote followers' well-being.

With concepts of constructive leadership behaviour like transformational leadership behaviour we would like to foster leaders' health oriented leadership behaviour as it's association with followers' well-being has been investigated ample. ${ }^{14}$ What needs to be added to previous research are more effective leadership intervention approaches in the workplace hospital with view to followers' mental health and the link of leaders' leadership behaviour to their own well-being. First studies showed mixed results. Zwingmann $e t a l^{11}$ reported a negative association of transformational leadership and leaders' emotional exhaustion. Kaluza et $a \hat{l}^{2}$ found constructive leadership to be related with leaders' work-related well-being in their meta-analytically approach.

In order to pilot this new stress-preventive leadership approach, we focused in this phase-II study on feasibility, acceptance and intrapersonal changes of participating leaders, measured by psychological outcomes as well as qualitative focus groups. Therefore, we asked the following research questions: (1) How do participants evaluate the feasibility and acceptance of the stress-preventive leadership intervention? (2) Do self-rated evaluation of work-related psychological stress, well-being and transformational leadership competency change in participants when measured before the intervention, after the last training session and after the intervention? (3) Has the intervention brought about a change in leaders' everyday work after participating in the intervention?

\section{METHODS \\ Study design and registration}

\section{Participants}

All leaders of middle management of the tertiary hospital with and without patient contact were invited to participate independently of their field of expertise. They were inclusively informed via email and could register themselves with the help of the hospital's Academy for Education and Personnel Development. Participants were given a written study information. After reading, informed written consent was obtained with the opportunity to withdraw their consent at any time. By withdrawing consent, participants' questionnaire data would be deleted. Participants were defined as drop outs for the 
analysis of psychological outcomes if they did not participate in the first module of the stress-preventive leadership intervention since this module set the groundwork for all the upcoming modules. Physicians and nurses received Continuing Medical Education(CME) credits for their participation.

\section{Patient and public involvement}

Prior to the leadership intervention 60 semistructured individual telephone interviews with 30 leaders of middle management and 30 employees without leadership position were conducted to discuss required content and format of a stress-preventive leadership intervention. ${ }^{33}$ The results of this needs assessment were included in the development of the intervention. Beyond that, there was no further involvement of potential participants or public.

\section{Intervention}

Stress-preventive leadership intervention

The intervention was developed based on expert knowledge, a systematic literature search, ${ }^{20}$ and a needs assessment. It took place in an interprofessional and interactive group setting and was conducted by two trainers (an educator and a psychologist). The intervention had a total duration of 24 hours, and was divided into five sessions. The first four sessions took place fortnightly and each covered a content module. The fifth session took place 3 months after the fourth session and covered a module for reflection and networking. While the first session lasted 8 hours, the sessions 2-4 lasted 4 hours each time. The first four sessions were supplemented by additional offerings (see figure 1). The intervention ran five times between 11 June 2018 and 3 March 2020. During this time, the whole intervention was repeated five times consecutively. A maximum of 20 leaders participated in each intervention group.

The intervention covered two main topics: the individual strain coping of leaders at the workplace hospital and the development of stress-preventive leadership attitude and behaviour based on the concept of transformational leadership. See table 1 for further details on ingredients of the intervention.

The contents were presented as short keynote presentations. Furthermore, extensive group and individual work took place to reflect participants' individual situation and to encourage interprofessional communication and networking. Additionally, participants received detailed written summaries at the beginning of every intervention module and memory cards with the main messages of the module at the end of each module. Short e-mail reminders were sent to the participants between the intervention modules with citations and short remarks to remind them of the intervention content in their everyday work.

\section{Data collection}

Psychological outcomes (irritation, well-being and transformational leadership) were measured pseudonymously. They were collected at the beginning of the intervention (T0), directly after module 4 (T1), and after a 3-month follow-up (T2). Feasibility and acceptance were collected anonymously directly after every module and concerning the whole intervention after module 5. Module 5 included qualitative focus group discussions to capture leaders' changes in every day work by participating in the intervention.

Figure 1 gives an overview of the timeline of the intervention and data collection.

\section{Quantitative variables}

Irritation (Irritation Scale)

The concept of irritation describes cognitive as well as emotional strain in the working context. Cognitive irritation is defined as the incapacity to switch off from work, and emotional irritation comprises an increase of negative interactions and irritability. ${ }^{34}$ Both constructs of cognitive and emotional strain in the working context are sensitive to change. Irritation was measured by the Irritation scale (IRR) ${ }^{34}$ which consists of eight items, with three items measuring cognitive irritation and five items assessing emotional irritation. Participants evaluated themselves on a seven-point Likert scale ranging

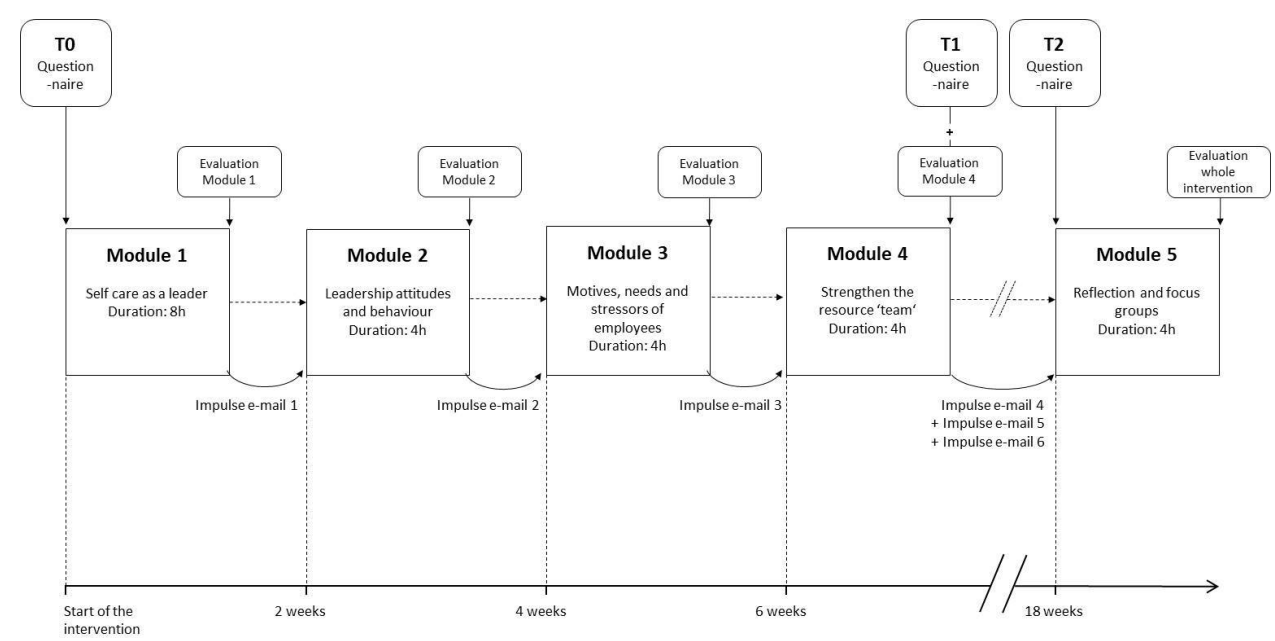

Figure 1 Intervention procedure. 
Table 1 Content of the stress-preventive leadership intervention

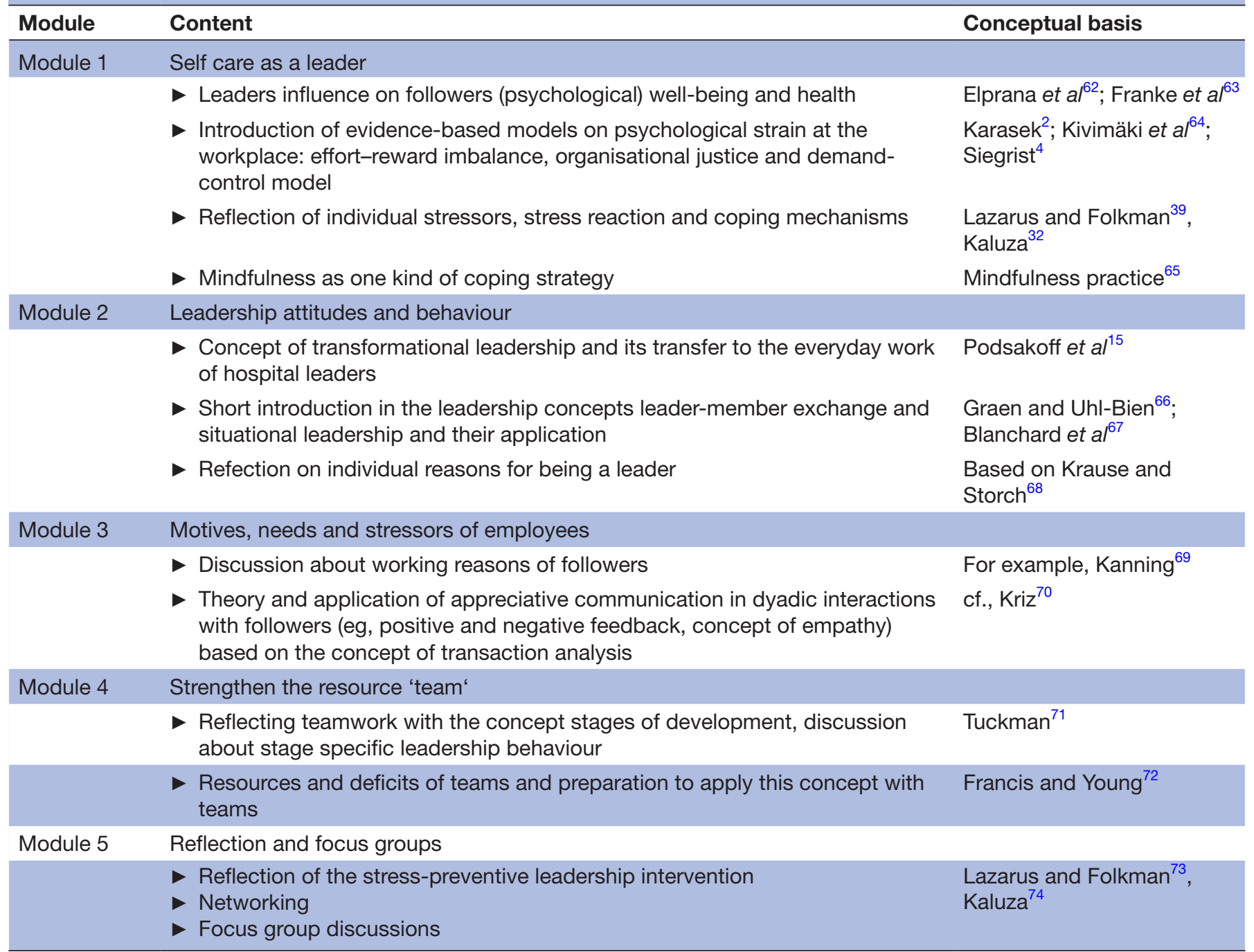

from 1 (strongly disagree) to 7 (strongly agree) with higher scores indicating higher irritation. The items were aggregated into two subscales, cognitive and emotional irritation. A previous study assessing the psychometric properties of the scale found internal consistency ranging between 0.83 and $0.90 .^{34}$ In this study, the subscales of cognitive and emotional irritation were calculated. Their internal consistency measured by Cronbach's alpha was $\alpha=0.89$ and $\alpha=0.89$, respectively.

\section{Well-being (WHO-5 Well-being Index)}

The concept of subjective well-being was implemented as a measurement of psychological health-related life quality. ${ }^{35}$ In this study, the WHO-5 questionnaire ${ }^{3637}$ was used to assess participants' subjective well-being during the previous 2 weeks. Participants responded to five items on a unidimensional six-point Likert scale ranging from 0 (at no time) to 5 (all the time). The ratings were aggregating to one percentage score. To qualify subjective wellbeing, the WHO-5 questionnaire is commonly scored as a percentage score, with $100 \%$ indicating the highest wellbeing, and lower percentages a lower sum respectively.
The WHO-5 questionnaire shows a high internal consistency with Cronbach's $\alpha=0.92 .{ }^{36}$ Internal consistency in this study was $\alpha=0.87$.

Transformational leadership (Questionnaire on Integrative Leadership)

To measure participants' subjective evaluation of their transformational leadership behaviour, module A of the Questionnaire on Integrative Leadership (Fragebogen zur Integrativen Führung, FiF), ${ }^{16}$ with six scales (Individuality, Vision, Role Modelling, Innovation, Team Sprit and Performance development) was applied. Participants rated their own leadership style on 24 items on a fivepoint Likert scale, ranging from 1 (strongly disagree) to 5 (strongly agree). Ratings were summed up to one overall average score. Internal consistency ranged between $\alpha=0.75$ and $\alpha=0.83{ }^{16}$ In this study, internal consistency for transformational leadership behaviour was $\alpha=0.91$.

Feasibility and acceptance

Feasibility and acceptance of the stress-preventive leadership intervention were measured by a self-developed 
intervention specific questionnaire and assessed after module 1 to module 4 . After module 5, participants assessed their satisfaction, their recommendation and the practical relevance of the whole intervention (original questions see online supplemental material).

\section{Qualitative data \\ Focus groups}

An accompanying qualitative evaluation of the stresspreventive leadership intervention was carried out through focus group discussions in the fifth module. All participants had the opportunity to discuss perceived changes in their leadership behaviour as a result of the intervention. Participants were asked: What has changed for yourself as a result of the intervention 'stress-preventive leadership in the hospital'? How has it changed? Are there example situations? This question route was part of seven main question routes regarding changes after participation in the intervention (changes for leaders, changes for followers and effectiveness in the leadership role) and questions on the reflection of the contents of the intervention (reflection on the concept of stress-preventive leadership, the implementation of contents learnt and related barriers). Results of all question routs especially perceived limits and potentials of the implementation of a stress-preventive leadership intervention are reported elsewhere. ${ }^{33} \mathrm{~S}=10$ focus groups were conducted in two parts each, with a 10 min break after about $45 \mathrm{~min}$, followed by another exchange for about 45 min. Per focus group, between 5 and 7 participants were discussing.

\section{Quantitative analysis}

For the description of participants, feasibility and acceptance the arithmetic mean (M), the standard deviation (SD), the range (range) and percentage values were used. For psychological outcomes linear mixed models were calculated to account for the internal time-dependent structure of the data. Analyses were conducted with $\mathrm{R}$ and R studio. ${ }^{38}{ }^{39}$ We fitted the data with the restricted maximum likelihood criterion and included a random intercept for each participant to account for level-1 variance between participants. Linear mixed model warrants analyses even for cases with missing values. This way, we could keep participants in the sample even if some of their data was missing. For each of the four outcome variables-cognitive irritation, emotional irritation, well-being and transformational leadership-we fitted a linear mixed model. The fixed effects were the different measurement points as a categorical variable. Linear mixed effect models were estimated using the software from the lme 4 and lmerTest packages. ${ }^{4041}$

\section{Qualitative analysis}

The focus group discussions were recorded and transcribed verbatim ${ }^{42}$ and anonymised simultaneously. The MAXQDA software was used to organise the data during analysis. ${ }^{43}$ Data material was analysed using
Table 2 Participants' age separated in age groups

\begin{tabular}{lcc}
\hline & \multicolumn{2}{l}{ Participants } \\
\cline { 2 - 3 } Age groups in years & $\%$ & $\mathbf{n}$ \\
\hline $25-30$ & 9.1 & 8 \\
$31-35$ & 14.8 & 13 \\
$36-40$ & 22.7 & 20 \\
$41-45$ & 19.3 & 17 \\
$46-50$ & 14.8 & 13 \\
$51-54$ & 15.9 & 14 \\
$>55$ & 3.4 & 3 \\
\hline
\end{tabular}

$\mathrm{n}=5$ participants didn't provide information on their age, $\mathrm{N}=93$ leaders participating in at least one session.

qualitative content analysis. ${ }^{44}$ While the first transcripts $(s=4)$ of the focus group discussions were completely coded, the remaining transcripts $(s=6)$ of the discussions were completely reviewed, but only new content was coded and used for further analysis. The data were coded using deductive categories set including category definitions, anchor examples and coding rules. ${ }^{45}$ Then the paraphrasing of the coded contents and the abstraction of the paraphrases using generalisations were performed. By abstraction of the paraphrases, the data material was reduced and further structured. The analysis steps of coding, paraphrasing and abstracting were carried out by at least two persons to support intersubjectivity. ${ }^{46}$ Researchers from the disciplines of sociology and psychology conducted the analysis.

\section{RESULTS}

\section{Participants}

$\mathrm{N}=93$ leaders of the middle management of a tertiary hospital in southern Germany participated in $\mathrm{k}=5$ stresspreventive leadership interventions. Of those, 49 leaders identified themselves as female, 39 as male and 5 did not disclose information on their gender. For an overview of participants' age distribution, see table 2. Table 3 provides information on the professional background of the participants. Participants average number of years in a hospital leadership position was $\mathrm{M}=5.57$ years $(\mathrm{SD}=6.14$, range $=0-30, n=88$ ) and their average number of followers was $\mathrm{M}=25,(\mathrm{SD}=30$, range $=2-180, \mathrm{n}=86)$. Since the intervention took place during participants' working hours, there were participants who could not participate in single sessions of the intervention due to clinical obligations, illness or holiday. The total amount of participants per session were for session $1 \mathrm{n}=88$, session $2 \mathrm{n}=64$, session $3 \mathrm{n}=67$, session $4 \mathrm{n}=69$, session $5 \mathrm{n}=64$. Five participants were excluded to further quantitative analysis of psychological outcomes because they did not participate in the first session of the intervention. Additionally, a subgroup of $n=60$ participants participated in one of 10 semistructured focus group discussions in session 5 . 
Table 3 Participants' professional background

\begin{tabular}{lrr} 
& \multicolumn{2}{l}{ Leaders } \\
\cline { 2 - 3 } Professional background & $\%$ & $\mathbf{n}$ \\
\hline Physicians & 30.9 & 29 \\
\hline Nursing sector & 24.5 & 23 \\
\hline Therapeutic professionals & 9.6 & 9 \\
\hline Adminstration & 12.8 & 12 \\
\hline Information technology (IT) & 3.2 & 3 \\
\hline Clinical services & 5.3 & 5 \\
Scientists & 1.1 & 1 \\
\hline Others & 7.4 & 7 \\
\hline
\end{tabular}

n, number of participating leaders; $n=4$ leaders did not provide information on their professional field, $\mathrm{N}=93$ leaders participating in at last one session.

\section{Feasibility}

After the intervention, participants assessed the feasibility of the whole leadership intervention concept. All participants were satisfied or very satisfied with the stresspreventive leadership intervention and rated the intervention as very practical relevant or practical relevant $(100 \%, \mathrm{n}=64) .92 .1 \% \quad(\mathrm{n}=59)$ would recommend the intervention. For further information on the modulespecific evaluation, see table 4 .

\section{Psychological outcomes}

Overall, significant improvements were found for cognitive irritation, well-being, and transformational leadership. The development of the average scores across these four indices can be seen in figure 2 .

To estimate the change in irritation, well-being and transformational leadership, we regressed those scores against time nested within individuals. The results of the linear mixed models of all indices can be seen in table 5 . The table depicts the size of the fixed effect as well as its statistical significance based on Satterthwaite's estimation of df. ${ }^{47}$ Error variance and variance of the random effects are reported in the lower half of table 5 .

Effect sizes of the fixed effects were relatively small, most of the variance was explained by differences between
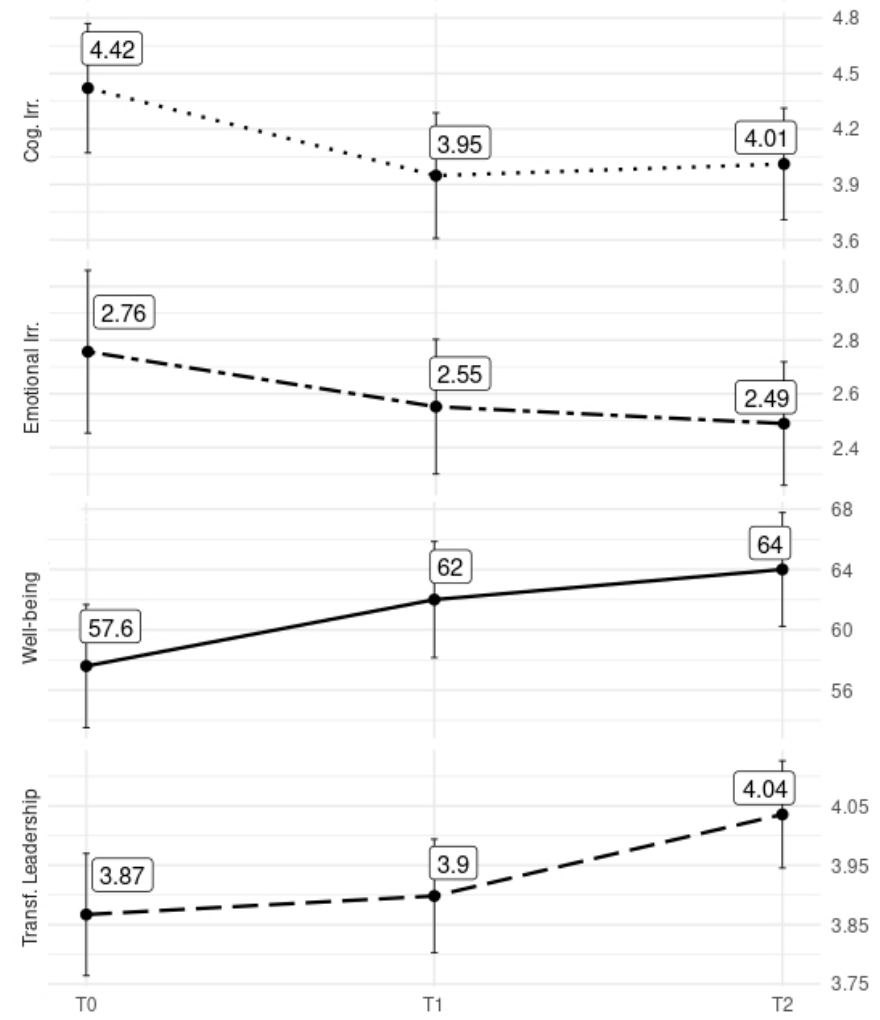

Figure 2 Development of average scores of irritation and emotional irritation, well-being and transformational leadership across the measurement points T0, T1, T2. Axes on the right show corresponding scale values. Bullets mark the mean values, whiskers the corresponding SE of the mean. Exact mean values are mentioned in the text boxes.

individuals, as can be seen in the overall high model fits Conditional $\mathrm{R}^{2}{ }^{48}$ The proportion of variance explained by the fixed effect, that is, the change within time, is expressed in the Marginal $\mathbf{R}^{2}$ value. For the significant fixed effects, the variance explained by the time variable is around $2 \%$. The observed change in the outcome variables is thus significant but small.

Irritation (IRR)

Cognitive irritation significantly decreased over time, the most pronounced change occurred between baseline (T0)

Table 4 Module-specific evaluation of participants

\begin{tabular}{|c|c|c|c|c|c|c|c|c|c|c|c|c|c|c|c|}
\hline \multirow[b]{3}{*}{ Modules } & \multicolumn{5}{|c|}{ Satisfaction } & \multicolumn{5}{|c|}{ Practical relevance } & \multicolumn{5}{|c|}{ Recommendation } \\
\hline & ++ & + & - & $\mathbf{n}$ & $\mathbf{m}$ & ++ & + & - & $\mathbf{n}$ & $\mathbf{m}$ & ++ & + & - & $\mathbf{n}$ & $\mathbf{m}$ \\
\hline & $\%$ & & & & & $\%$ & & & & & $\%$ & & & & \\
\hline Module 1 & 57.5 & 40.2 & 1.1 & 86 & 1 & 42.5 & 54.0 & 2.3 & 86 & 1 & 72.4 & 25.3 & 2.3 & 87 & 0 \\
\hline Module 2 & 53.1 & 43.8 & 3.1 & 64 & 0 & 54.7 & 42.2 & 3.1 & 64 & 0 & 73.4 & 26.6 & 0 & 64 & 0 \\
\hline Module 3 & 83.6 & 16.4 & 0 & 67 & 0 & 80.6 & 17.9 & 1.5 & 67 & 0 & 85.1 & 14.9 & 0 & 67 & 0 \\
\hline Module 4 & 50.7 & 43.5 & 5.8 & 69 & 0 & 53.6 & 42.0 & 4.3 & 69 & 0 & 47.8 & 18.8 & 2.9 & 48 & 21 \\
\hline
\end{tabular}

$\%$, presented percentage values; ++, the proportion of all participants who strongly agreed to the questions or statements; +, proportion of all participants who agreed to the questions or statements; -, proportion of all participants who tended to disagree; $n$, number of participants who answers to question; $\mathrm{m}$, number of missing values. 
and the assessment after the forth module (T1), while the irritation score remained relatively stable after the forth module (T1) and follow-up before the fifth module (T2). The change of emotional irritation did not reach statistical significance. Descriptively, the average emotional irritation dropped from baseline to the timepoint after module 4, but individual trends were heterogeneous and the effect size was too small to detect the effect statistically. Results for the linear regression models can be seen in table 5 , columns 1 and 2 .

Well-being (WH0-5 Well-being Index)

Subjective well-being improved significantly for the participants in the sample of the stress-preventive intervention at T2 and increased across the three measurement points, cf. column 3 of table 5 for the results of the linear mixed model.

\section{Transformational leadership (Questionnaire on Integrative} Leadership)

Self-rated transformational leadership increased from baseline to follow-up significantly the most pronounced change of average score occurred between data assessment after module 4 (T1) and follow-up (T2); cf. column 4 of table 5 for the results of the linear mixed model.

\section{Focus groups}

Participants reported changes in their everyday work due to their participation in the intervention concerning their individual strain coping, their positive interaction within their team, their communication as leaders and their awareness of stress at the workplace in general. Leaders reported changes in knowledge/sensitivity, attitude and behaviour.

\section{Individual strain coping}

Participants reported that the intervention helped them to achieve a higher sensitivity concerning their own strain experience (eg, personal limits and resources) and the detection of leadership-specific stressors. Some participants felt strengthened and more motivated concerning their leadership tasks and reported a more tolerant attitude towards themselves as leaders. Since the start of the intervention, some leaders would practice more stresspreventive and strain-reducing behaviour. Practising mindfulness to reduce working speed, taking breaks and considered decision making were mentioned most frequently.

The mindfulness and I partly succeeded in limiting the depletion of my own resources and to evaluate and handle everything else in a competent manner. For example, it was very useful to me to analyze, what drives me. Only having an understanding for that, even if I can't change the situation immediately, helps me to evaluate things different and also to have more positive emotions in my everyday work or regarding stages of projects and to have a more relaxed view on the processes helps me enormous. (8934404-PO-01_20190228_135716) 
Positive interaction within the team

Participants commented on team development and succeeded teamwork between leaders and teams. Leaders would have expanded their knowledge on stressors and resilience regarding followers, team culture and the function of a leader within a team. Some participants would have the idea to involve followers to a greater extent in problem-solving. Furthermore, they mentioned a greater degree of trust in their followers, a higher flexibility in task processing and a higher acceptance concerning unchangeable working conditions. Concerning their leadership behaviour participants would have tried to reduce the transfer of their own stress to their followers. Moreover, leaders would feel more responsible to solve conflicts between followers; they would prioritise requests from followers more strongly, would distribute working tasks more thoughtfully within the team and would try to foster the interaction within and their own transparency towards their team.

Well, for myself changed, for example, that I involve my staff members more in my tasks or topics. Even if they don't have to evaluate the topics themselves. But, just so they know, what is happening or which topics I am dealing with, if I don't have time. And actually I want them to know, because they bring up aspects that add to the topic - or whatever - and that's why it is very important to me to communicate and also that there is good exchange of ideas. Yes. (7517680-PO01_Auftrag_05.11.18_20181015_140547).

\section{Communication as leaders}

Participants reported on a greater knowledge and sensitivity concerning communication techniques and processes. This would be reflected in a more empathetic and clearer attitude in discussions. On a behavioural level, this would lead to more frequent team meetings and communication with followers. Participants would try to listen more actively. In team meetings, the concerns and perceived strain of followers would be given more space and participants would strive for more frequent, intimate and positive feedback.

As well in meetings, for example with representatives of departments, I have made it my ambition to be the last to leave the room after the meeting, for example. Because I think, that's also part of it. Before, I often was the first to leave, in a manner like: Now we are finished, all good. Keep up the good work. And I hurried out the room to my office (laughs). And now I am the last and I am more aware of what some may have or don't have to say. (9487813-PO01_Auftrag_05.05.20_20000102_084927)

\section{Awareness of stress at the workplace in general}

Participants mentioned being more aware of stress and illness at work in general, working structures that cause mental illness (eg, communication deficits) and workrelated mental illnesses of followers.

Also, I noticed, that I am concentrating more on the topic disease and health. Also, I would say, what I noticed before, oh, he is not doing so well at the moment or he seems to be stressed, but the simple thought, that it is something that over a longer period of time makes people sick, that thought didn't accrue to me most of the times to be honest. (7517680-PO01_Auftrag_05.11.18_20181015_140547)

\section{DISCUSSION}

\section{Main findings}

The stress-preventive leadership intervention has been evaluated as highly satisfying, application-oriented and participants would recommend the intervention. Furthermore, ratings of cognitive irritation, well-being and transformational leadership changed significantly over time. Cognitive irritation scores were significantly reduced at $\mathrm{T} 1$ and persisted over time. Well-being scores increased across all three measurement points and transformational leadership scores increased from baseline (T0) to follow-up (T2). No significant changes could be detected in emotional irritation. In the focus group discussions, participants reported an improvement concerning their own strain coping, changes in their way to cooperate and develop relationships with followers, a higher awareness for communication techniques and appreciative feedback culture as well as a higher awareness of mental health in the workplace hospital by participating in the intervention.

\section{Feasibility}

With regard to participants' ratings on satisfaction, practical relevance and recommendation, module 3 was rated particularly well with regard to all indices. This could be due to didactical or content-related aspects. Module $3 \mathrm{had}$ the highest proportion of exercises in small groups and partner work. Participants had time to get in contact and strengthen interprofessional contacts. Moreover, the evaluation could emphasise the need for more peer-assisted learning for leaders generally. Peer-assisted learning is already used successfully with medical students ${ }^{49}$ and in other contexts of academic medicine. ${ }^{50}$ It could be extended to stress-preventive leadership approaches, for example, in form of regular intervision groups or peer to peer coaching as Gabbe $e t a l^{25}$ conducted a mentoring approach for new chairs of medical departments in their pilot study. Although they could not report a beneficial effect of their mentoring programme, they emphasised the need of mentoring programmes at the respective workplace. In module 3 , participants dealt with dyadic communication by practicing active listening, giving critical and positive feedback in an appreciative way based on situations of their everyday work in the hospital context. According to participants' positive evaluation, refreshing 
basic communication skills seems important. This should be taken into account when developing future leadership interventions for the healthcare sector.

\section{Psychological outcomes}

The intrapersonal reduction of perceived cognitive irritation and the improvement of perceived well-being in hospital leaders in this study are in line with results of other stress-preventive leadership interventions in the healthcare sector. Haraway and Haraway ${ }^{21}$ found in their pre-post study a significant reduction of intrapersonal occupational strain from before the intervention to 1 month after the intervention. Luk ${ }^{22}$ reported a significant intrapersonal improvement of leaders' work-related well-being after the intervention compared with before the intervention.

In our study, the strongest reduction of average scores of cognitive irritation was observed between $\mathrm{T} 0$ and $\mathrm{T} 1$. Thus, participants reported a psychological strain reduction directly after the intervention, which remained stable until T2. When evaluating the effects of a stress-preventive intervention, it is important to consider its long-term effect. Ideally, beneficial training effects prevail, rather than fading quickly after the training. A 3-month stability of the observed improvements of relevant psychological outcome variables might be interpreted as a hint that potential effects do not cease instantly. A psychological strain reduction post-intervention has been observed in other person directed stress-preventive interventions as well. In their review, Awa $e t a{ }^{\tilde{1}}$ examined the effect of interventions on burnout-related symptoms. They reported a reduction of burn-out symptoms lasting until a 6-month follow-up in $82 \%$ of person-directed interventions but significant long-term effect over a period of 1 year on burn-out-related symptoms has been shown only in the study of Rowe. ${ }^{52}$ In this long-term study, Rowe ${ }^{52}$ conducted an intervention to reduce burn-out symptoms and reported sustained lower scores for burnout over 2.5 years, when participants got short intervention modules for refreshment at 5 months, 11 months and 17 months after their intervention. Thus, interventions with continuous training sessions over a longer period of time could extend the stress-preventive effect. To examine the long-term effects of stress-preventive leadership interventions more controlled long-term studies are needed with longer follow-up periods. If effects could be confirmed, stress prevention skills should be integrated as a regular part of leadership development as leaders need to train strain coping skills exactly like other human resource management skills. This statement is additionally supported by the result that leaders' own strain is negatively related to stress-preventive leadership behaviour. ${ }^{53}$ One future-oriented example for an extensive leadership development programme with parts of stress-preventive leadership behaviour like for example, emotional intelligence or conflict resolution is the leadership development programme at Cleveland Clinic. ${ }^{54}$
Besides the promising changes in psychological outcomes, participants reported higher scores on transformational leadership behaviour from baseline (T0) to follow-up (T2). This result points to an improvement in transformational leadership through the intervention under study here. This result is in line with the results of other studies in the healthcare sector. Saravo et $a \tilde{l}^{5}$ reported a significant improvement of transformational leadership after an intervention in the self-assessment of leaders and in the assessment by an external evaluator compared with before the intervention. For organisations, especially tertiary hospitals, it would be interesting to analyse if the steady changes in transformational leadership can impact the organisational culture. ${ }^{56}$ If the improvement in transformational leadership steadily changes the organisational culture, it could be possible that it has a positive impact on role behaviour, such as in-role and extra-role behaviors ${ }^{57}$ that would help hospitals in dealing with the growing pressure. However, so far research on the mechanisms that explain the long-term stability of the change in leading behaviour is lacking and should be addressed in future research.

In addition to that, the largest increase of average scores of transformational leadership over time was observed between $\mathrm{T} 1$ and T2. This observation could point to a delayed improvement of transformational leadership. This delayed development has also been reflected in the study of Abrell et al. ${ }^{58}$ Followers' assessment revealed an improved transformational leadership style of their leaders not 3 months but 6 months after participating in a leadership intervention. This could be due to the fact that behavioural changes need time to be implemented in everyday work. Future intervention studies could examine transformational leadership in a controlled design with the help of manifold feedback sources (eg, followers, external evaluators) to assess transformational leadership in a more valid way for example, see Saravo $e t$ $a l^{55}$

When the observed changes in psychological outcomes and transformational leadership behaviour are considered together, we could underpin the hypothesis that leading in a transformational way benefits from leaders' improved mental health. Research has shown that transformational leadership is a psychologically demanding leadership style and needs psychological resources as transformational leadership behaviour can increase emotional exhaustion of leaders over time. ${ }^{31}$ Furthermore, Byrne et a $\tilde{l}^{9}$ could show that leaders' mental ill-health was negatively related to their transformational leadership behaviour and Lange $e t a l^{60}$ reported a positive association between leaders' mindfulness and their transformation leadership behaviour. This highlights once again the need of good stress coping skills for hospital leaders. In addition, research should look at the overall ratio of costs and gains of transformational leadership for the leader, as the described evidence for both the demands as well as the benefits of such leadership behaviour should be taken into account. Future research should analyse under what 
conditions the costs can outweigh the gains, as contextual elements seem to impact whether the leader perceives exhaustion over time ${ }^{31}$ or not. Especially the workplace hospital can vary with regard to the demand and control in general, ${ }^{12}$ but specifically when burdened by acute health crises. Such aspects of the everyday work could explain the diametral effect of leading transformationally.

\section{Focus groups discussions}

With the help of focus group discussions, participants gave an insight in changes they made in their everyday leadership after having received the intervention. Such qualitative approaches were also used by other stresspreventive leadership interventions in form of essays ${ }^{22}$ or open questions before and after the intervention. ${ }^{21}$ The results of our focus group discussions showed that the intervention improved participants' knowledge of stresspreventive leadership and also affected their leadership values and behaviour which are important for sustainable changes in their everyday work as a leader. Through the focus groups discussions the psychological way in which the intervention contributed to these changes became clear. This supports the assumption that the intervention contributed to leaders' reduced irritation, improved well-being and improved transformational leadership. With the help of further qualitative approaches, future research has the opportunity to examine leadership change processes in detail. This would help to get a better understanding of what motivates and supports leaders to act in a stress-preventive way.

\section{Strengths and limitations}

This mixed-method phase-II study evaluated an evidencebased stress-preventive leadership intervention for middle management adapted to the highly psychologically demanding workplace hospital. It added quantitative and qualitative evidence on its' feasibility and practical use. Due to the uncontrolled study design and the voluntary participation, no statement can be made about the effectiveness of the intervention. In addition, the study results are only based on self-disclosure.

\section{CONCLUSION}

We developed a new stress-preventive leadership intervention for middle management in the workplace hospital. It contained an innovative combination of strain preventive concepts for leaders and constructive stress-preventive leadership behaviour concepts. Study results show participants' perceived reduction in work-related strain and improvement in well-being and transformational leadership after the intervention. In focus group discussions participants could describe changes in leadership behaviour and values that they attributed to participating in the intervention. These qualitative results support the assumption of effectiveness of the intervention. This intervention format is worthwhile to be further investigated in a randomised controlled trial (as it currently is as one module of the SEEGEN-trial). ${ }^{61}$ Future studies should also capture the perspective of followers on transformational leadership and followers' work-related strain.

\section{Author affiliations}

${ }^{1}$ Department of Psychosomatic Medicine and Psychotherapy, University Hospital Tuebingen, Tuebingen, Germany

${ }^{2}$ Institute of Occupational and Social Medicine and Health Services Research, University Hospital Tuebingen, Tuebingen, Germany

${ }^{3}$ Echt:Zeit Coaching, Esslingen, Germany

${ }^{4}$ Department of Psychosomatic Medicine and Psychotherapy, Ulm University Medical Center, Ulm, Germany

${ }^{5}$ Institute of Psychology, Work and Organisational Psychology, University of Duisburg-Essen, Essen, Germany

${ }^{6}$ Institute of Occupational, Social and Environmental Medicine, Centre for Health and Society, Faculty of Medicine, University of Duesseldorf, Duesseldorf, Germany

${ }^{7}$ Department for General Internal Medicine and Psychosomatics, University Hospital Heidelberg, Heidelberg, Germany

${ }^{8}$ Chair of Business Administration, Human Resource Management and Organisation Studies, Heinrich Heine University Duesseldorf, Duesseldorf, Germany

${ }^{9}$ Psychiatry and Psychotherapy II, Ulm University, Ulm, Germany

${ }^{10}$ Department of Psychosomatic Medicine and Psychotherapy, Otto von Guericke University Magdeburg, Magdeburg, Germany

Acknowledgements The stress-preventive leadership intervention is part of the multicentre project SEEGEN (SEElische GEsundheit am Arbeitsplatz KrankeNhaus). SEEGEN is a joint project, the success of which was made possible by good interdisciplinary cooperation. We would like to thank the members of the SEEGEN consortium as well as all participating hospital employees and especially the management and staff who supported us on site. We thank Dr. Dagmar Brendle and Katja Böttle (Academy for Education and Personnel Development of the University Hospital Tuebingen) for their support. Thanks also to our student assistants Charlotte Schenk, Antonia Seitz, and Natalia Radionova who supported the qualitative analysis. This study is part of the first author's (FS) work toward a doctoral degree. FS receives a PhD grant from the 'Konrad-Adenauer-Stiftung' (Konrad Adenauer Foundation). The work of the Institute of Occupational Medicine, Social Medicine and Health Services Research Tuebingen is supported by an unrestricted grant of the employers' association of the metal and electric industry Baden-Wuettemberg (Suewestmetall). And we acknowledge additional support by Open Access Publishing Fund of University of Tuebingen.

Contributors FS, TS-D, SuS, MAR and FJ developed the stress-preventive leadership intervention. FS, TS-D and FJ obtained the approval from the Ethics Committee of the Medical Faculty of the University Hospital Tuebingen. FS, TS-D and IA were responsible for the recruitment process, FS, TS-D and SuS designed the intervention material and organised the data collection. FS, TS-D and SS conducted the focus groups. ET conducted the qualitative analysis with the interdisciplinary team including FS, TS-D, FJ and MAR. ZRA, IA and FS conducted the quantitative analysis. FS drafted the manuscript with considerable contribution from ZRA, IA, TS-D, FJ, RE, NM, AM, PA, MH, CN, SR, BP, HG and MAR. All authors read and approved the final version of this manuscript. FJ supervised the project. FS is the guarantor.

Funding This project is funded by the German Federal Ministry of Education and Research (BMBF) under the funding code 01GL1752C.

Competing interests SuS is the owner of Echt-Zeit Coaching, Esslingen (Germany), and was involved in the development and implementation of the intervention sessions together with FS and TS-D as part of cooperation with the University Hospital Tuebingen.

Patient consent for publication Not applicable.

Ethics approval The study was approved by the ethics committee of the University Hospital and the Medical Faculty of Tuebingen (208/2018B01) and was carried out in accordance with the recommendation of the ICH-GCP-guidelines, Declaration of Helsinki.

Provenance and peer review Not commissioned; externally peer reviewed.

Data availability statement No data are available. No additional data are available. The Chief Executive Board and the Employees' Council of the tertiary hospital had to approve the implementation of this study. This approval required that raw data be made available only to direct project members. 
Supplemental material This content has been supplied by the author(s). It has not been vetted by BMJ Publishing Group Limited (BMJ) and may not have been peer-reviewed. Any opinions or recommendations discussed are solely those of the author(s) and are not endorsed by BMJ. BMJ disclaims all liability and responsibility arising from any reliance placed on the content. Where the content includes any translated material, BMJ does not warrant the accuracy and reliability of the translations (including but not limited to local regulations, clinical guidelines, terminology, drug names and drug dosages), and is not responsible for any error and/or omissions arising from translation and adaptation or otherwise.

Open access This is an open access article distributed in accordance with the Creative Commons Attribution Non Commercial (CC BY-NC 4.0) license, which permits others to distribute, remix, adapt, build upon this work non-commercially, and license their derivative works on different terms, provided the original work is properly cited, appropriate credit is given, any changes made indicated, and the use is non-commercial. See: http://creativecommons.org/licenses/by-nc/4.0/.

\section{ORCID iDs}

Felicitas Stuber http://orcid.org/0000-0002-7503-3881

Tanja Seifried-Dübon http://orcid.org/0000-0001-5935-539X

Rebecca Erschens http://orcid.org/0000-0002-4433-9378

Sascha Ruhle http://orcid.org/0000-0001-7855-2647

Bernd Puschner http://orcid.org/0000-0002-2929-4271

\section{REFERENCES}

1 Von dem Knesebeck O, Klein J, Frie KG. Psychosoziale Arbeitsbelastungen bei chirurgisch tätigen Krankenhausärzten. Dtsch Arztebl Int 2010;107:248-53.

2 Karasek R. Low social control and physiological deregulation-the stress-disequilibrium theory, towards a new demand-control model. Scandinavian Journal of Work, Environment \& Health 2008;6:117-35.

3 Bauer J, Groneberg D. Ärztlicher Disstress-eine Untersuchung baden-württembergischer Ärztinnen und Ärzte in Krankenhäusern. Dtsch Med Wochenschr 2013;138:2401-6.

4 Siegrist J. Adverse health effects of high-effort/low-reward conditions. J Occup Health Psychol 1996;1:27.

5 Bernburg M, Vitzthum K, Groneberg DA, et al. Physicians occupational stress, depressive symptoms and work ability in relation to their working environment: a cross-sectional study of differences among medical residents with various specialties working in German hospitals. BMJ Open 2016;6:e011369.

6 The Lancet. Physician burnout: a global crisis. Lancet 2019;394:93.

7 Badura B, Ducki A SH, et al. Fehlzeiten-Report 2018: Sinn erleben Arbeit und Gesundheit. Berlin: Springer, 2018.

8 Sanghera J, Pattani N, Hashmi Y, et al. The impact of SARS-CoV-2 on the mental health of healthcare workers in a hospital setting-A systematic review. J Occup Health 2020;62:e12175.

9 Degen C, Li J, Angerer P. Physicians' intention to leave direct patient care: an integrative review. Hum Resour Health 2015;13:74.

10 Nielsen K, Nielsen MB, Ogbonnaya C, et al. Workplace resources to improve both employee well-being and performance: a systematic review and meta-analysis. Work Stress 2017;31:101-20.

11 Hall LH, Johnson J, Watt I, et al. Healthcare staff wellbeing, burnout, and patient safety: a systematic review. PLoS One 2016;11:e0159015.

12 Sturm H, Rieger MA, Martus P, et al. Do perceived working conditions and patient safety culture correlate with objective workload and patient outcomes: a cross-sectional explorative study from a German university hospital. PLOS ONE 2 019;14:e0209487.

13 Loerbroks A, Glaser J, Vu-Eickmann P, et al. Physician burnout work engagement and the quality of patient care. Occup Med 2017;67:356-62.

14 Montano D, Reeske A, Franke F, et al. Leadership, followers' mental health and job performance in organizations: A comprehensive metaanalysis from an occupational health perspective. J Organ Behav 2017;38:327-50

15 Podsakoff PM, MacKenzie SB, Moorman RH, et al. Transformational leader behaviors and their effects on followers' trust in leader, satisfaction, and organizational citizenship behaviors. Leadersh $Q$ 1990;1:107-42.

16 Rowold J, Poethke U. Fragebogen Zur Integrativen Führung. FIF. Bern: Hogrefe, 2017.

17 Arnold KA. Transformational leadership and employee psychological well-being: a review and directions for future research. J Occup Health Psychol 2017;22:381-93.

18 Tsutsumi A. Development of an evidence-based guideline for supervisor training in promoting mental health: literature review. $J$ Occup Health 2011;53:1-9.
19 Kuehnl A, Seubert C, Rehfuess E, et al. Human resource management training of supervisors for improving health and wellbeing of employees. Cochrane Database Syst Rev 2019;9:CD010905.

20 Stuber F, Seifried-Dübon T, Rieger MA, et al. The effectiveness of health-oriented leadership interventions for the improvement of mental health of employees in the health care sector: a systematic review. Int Arch Occup Environ Health 2021;94:203-20.

21 Haraway DL, Haraway WM. Analysis of the effect of conflictmanagement and resolution training on employee stress at a healthcare organization. Hosp Top 2005;83:11-17.

22 Luk LA. Assessment of a leadership enhancement program for nursing managers of an acute General Hospital in Hong Kong. Open Nurs J 2018;12:133-41.

23 Eastburg MC, Williamson M, Gorsuch R, et al. Social support, personality, and burnout in nurses. J Appl Soc Psychol 1994;24:1233-50.

24 Greenberg J. Losing sleep over organizational injustice: attenuating insomniac reactions to underpayment inequity with supervisory training in interactional justice. J Appl Psychol 2006;91:58.

25 Gabbe SG, Webb LE, Moore DE, et al. Can mentors prevent and reduce burnout in new chairs of departments of obstetrics and gynecology: results from a prospective, randomized pilot study. Am J Obstet Gynecol 2008;198:653.

26 Stansfeld SA, Kerry S, Chandola T, et al. Pilot study of a cluster randomised trial of a guided e-learning health promotion intervention for managers based on management standards for the improvement of employee well-being and reduction of sickness absence: GEM study. BMJ Open 2015;5:e007981.

27 Zimber A, Rudolf A, Teufel S. Arbeitsbelastungen in Der Altenpflege reduzieren: ein Trainingsprogramm für Mitarbeiter und Leitungskräfte. Zeitschrift für Gerontologie und Geriatrie 2001;34:401-7.

28 SAGE Publications Sage CA. A three-step model of stress management for health leaders. healthcare management forum. Los Angeles, CA: SAGE Publications Sage CA, 2018.

29 Harms PD, Credé M, Tynan M, et al. Leadership and stress: a metaanalytic review. Leadersh Q 2017;28:178-94.

30 Vincent-Höper S, Gregersen S, Nienhaus A. Do work characteristics mediate the negative effect of transformational leadership on impaired well-being? Zeitschrift für Arbeits- und Organisationspsychologie 2017;61:167-80.

31 Zwingmann I, Wolf S, Richter P. Every light has its shadow: a longitudinal study of transformational leadership and leaders' emotional exhaustion. J Appl Soc Psychol 2016;46:19-33.

32 Kaluza AJ, Boer D, Buengeler C, et al. Leadership behaviour and leader self-reported well-being: a review, integration and metaanalytic examination. Work Stress 2020;34:34-56.

33 Tsarouha E, Stuber F, Seifried-Dübon T, et al. Reflection on leadership behavior: potentials and limits in the implementation of stress-preventive leadership of middle management in hospitals a qualitative evaluation of a participatory developed intervention. Journal of Occupational Medicine and Toxicology 2021;16:1-14.

34 Mohr G, Rigotti T, Müller A. Irritation-ein instrument Zur Erfassung psychischer Beanspruchung Im Arbeitskontext. Skalen-und Itemparameter AUS 15 Studien. Zeitschrift für Arbeits-und Organisationspsychologie A\&O 2005;49:44-8.

35 Schumacher J, Klaiberg A, Brähler E. Diagnostik von Lebensqualität und Wohlbefinden - Eine Einführung. In: Schumacher J, Klaiberg A, Brähler E, eds. Diagnostische Verfahren zu Lebensqualität und Wohlbefinden. Göttingen: Hogrefe, 2003.

36 Brähler E, Mühlan H, Albani C, et al. Teststatistische Prüfung und Normierung Der deutschen Versionen des EUROHIS-QOL Lebensqualität-Index und des WHO-5 Wohlbefindens-Index. Diagnostica 2007;53:83-96.

37 Topp CW, Østergaard SD, Søndergaard S, et al. The WHO-5 well-being index: a systematic review of the literature. Psychother Psychosom 2015;84:167-76.

38 R Foundation for Statistical Computing. R: A Language and Environment for Statistical Computing [program. Vienna, Austria, 2020.

39 RStudio. Integrated Development for $R$ [program. Boston, MA: RStudio, PBC, 2020.

40 Bates D, Mächler M, Bolker B, et al. Fitting Linear Mixed-Effects Models Using Ime4. J Stat Softw 2015;67:48.

41 Kuznetsova A, Brockhoff PB, Christensen RHB. ImerTest Package: Tests in Linear Mixed Effects Models. J Stat Softw 2017;82:26.

42 Dresing T, Pehl T. Praxisbuch Interview, Transkription \& Analyse. Anleitungen und Regelsysteme für qualitativ Forschende [Research in practice: interviews, transcription \& analysis. Instructions and control systems for qualitative researchers. Marburg dr dresing \& pehl $\mathrm{GmbH}, 2018$.

43 VERBI Software. MAXQDA. Berlin, Germany: VERBI Software, 2018. 
44 Mayring P, Fenzl T. Qualitative Inhaltsanalyse [Qualitative content analysis]. In: Handbuch Methoden der empirischen Sozialforschung [Handbook Methods of Empirical Social Research. Wiesbaden: Springer VS, 2014: 543-56.

45 Mayring P. Qualitative content analysis: demarcation, varieties, developments. Forum Qual Soc Res 2019;20.

46 Kruse J. Qualitative Interviewforschung. Ein integrativer Ansatz [Qualitative interview research. An integrative approach. Weinheim: Beltz Juventa, 2014

47 Luke SG. Evaluating significance in linear mixed-effects models in R. Behav Res Methods 2017;49:1494-502.

48 Nagelkerke NJD. A note on a general definition of the coefficient of determination. Biometrika 1991;78:691-2.

49 Tai J, Molloy E, Haines T, et al. Same-level peer-assisted learning in medical clinical placements: a narrative systematic review. Med Educ 2016;50:469-84.

50 Pololi L, Knight S. Mentoring faculty in academic medicine. A new paradigm? J Gen Intern Med 2005;20:866-70.

51 Awa WL, Plaumann M, Walter U. Burnout prevention: a review of intervention programs. Patient Educ Couns 2010;78:184-90.

52 Rowe MM. Skills training in the long-term management of stress and occupational burnout. Curr Psychol 2000;19:215-28.

53 Diebig M, Poethke U, Rowold J. Leader strain and follower burnout: exploring the role of transformational leadership behaviour. German Journal of Human Resource Management 2017;31:329-48.

54 Christensen T, Stoller JK. Physician leadership development at Cleveland clinic: a brief review. Australas Psychiatry 2016;24:235-9.

55 Saravo B, Netzel J, Kiesewetter J. The need for strong clinical leaders - Transformational and transactional leadership as a framework for resident leadership training. PLOS One 2017;12:e0183019.

56 Sow M. The relationship between leadership style, organizational culture, and job satisfaction in the US healthcare industry. Manag Econ Res J 2017:2017:1332.

57 Xenikou A. Transformational leadership, Transactional contingent reward, and organizational identification: the mediating effect of perceived innovation and goal culture orientations. Front Psychol 2017;8:8.

58 Abrell C, Rowold J, Weibler J, et al. Evaluation of a long-term transformational leadership development program. German Journal of Human Resource Management 2011;25:205-24.

59 Byrne A, Dionisi AM, Barling J, et al. The depleted leader: The influence of leaders' diminished psychological resources on leadership behaviors. Leadersh Q 2014;25:344-57.
60 Lange S, Bormann KC, Rowold J. Mindful leadership: mindfulness as a new antecedent of destructive and transformational leadership behavior. Gr Interakt Org 2018;49:139-47.

61 Mulfinger N, Sander A, Stuber F, et al. Cluster-randomised trial evaluating a complex intervention to improve mental health and wellbeing of employees working in hospital - a protocol for the SEEGEN trial. BMC Public Health 2019;19:1694.

62 Elprana G, Felfe J, Franke F. Gesundheitsförderliche Führung diagnostizieren und umsetzen. In: Felfe J, van Dick R, eds. Handbuch Mitarbeiterführung: Wirtschaftspsychologisches Praxiswissen für Fach- und Führungskräfte. Berlin: Springer, 2016: 147-56.

63 Franke F, Ducki A, Felfe J. Gesundheitsförderliche Führung. In: Felfe J, ed. Trends in Der psychologischen Führungsforschung. Göttingen: Hogrefe, 2015: 253-64.

64 Kivimäki M, Elovainio M, Vahtera J, et al. Organisational justice and health of employees: prospective cohort study. Occup Environ Med 2003;60:27-34.

65 Irving JA, Dobkin PL, Park J. Cultivating mindfulness in health care professionals: a review of empirical studies of mindfulnessbased stress reduction (MBSR). Complement Ther Clin Pract 2009;15:61-6.

66 Graen GB, Uhl-Bien M. Relationship-based approach to leadership: development of leader-member exchange (LMX) theory of leadership over 25 years: applying a multi-level multi-domain perspective. Leadersh Q 1995;6:219-47.

67 Blanchard KH, Blanchard M, Carew D. Leading at a higher level Situational Leadership II: The integrating concept. Upper Saddle River, NJ: Prentice Hall, 2007: 76-89.

68 Krause F, Storch M. Ressourcen aktivieren mit dem Unbewussten: Die Züricher Ressourcen Modell Bildkartei Bildformat DIN A6. In: 2. aktualisierte. Bern: Hogrefe, 2017.

69 Kanning UP. IEA, Inventar Zur Erfassung von Arbeitsmotiven. Bern: Hogrefe, 2016.

70 Kriz J. Grundkonzepte Der Psychotherapie. Weinheim: Beltz, 2014.

71 Tuckman BW. Developmental sequence in small groups. Psychol Bull 1965;63:384-99.

72 Francis D, Young D. Mehr Erfolg Im team. Hamburg: Windmühle, 1996.

73 Lazarus RS, Folkman S. Stress, appraisal, and coping. New York: Springer, 1984.

74 Kaluza G. Stressbewältigung: Trainingsmanual Zur psychologischen Gesundheitsförderung. 4., korrigierte ed. Berlin: Springer, 2018. 